\title{
Detecting Tipping points in Ecological Models with Sensitivity Analysis
}

\author{
G.A. ten Broeke ${ }^{1 *}$, G.A.K. van Voorn ${ }^{1}$, B.W. Kooi $^{2}$, J. Molenaar ${ }^{1}$ \\ ${ }^{1}$ Biometris, Wageningen University \& Research, the Netherlands \\ ${ }^{2}$ Faculty of Earth and Life Sciences, VU University, the Netherlands
}

\begin{abstract}
Simulation models are commonly used to understand and predict the development of ecological systems, for instance to study the occurrence of tipping points and their possible ecological effects. Sensitivity analysis is a key tool in the study of model responses to changes in conditions. The applicability of available methodologies for sensitivity analysis can be problematic if tipping points are involved. In this paper we demonstrate that not considering these tipping points may result in misleading statistics on model behaviour. In turn, this limits the applicability of simulation models in ecological research. Tipping points are best revealed when asymptotic model behaviour is considered, i.e. by applying bifurcation analysis. Bifurcation analysis, however, is limited to deterministic dynamic models, whereas many ecological simulation models are nondeterministic and can only be analysed using sensitivity analysis methodologies. In this paper we explore the possibilities for applying methodologies of sensitivity analysis to analyse models with tipping points. The Bazykin-Berezovskaya model, a deterministic ecological model of which the structure regarding tipping points is known a priori, is used as case study. We conclude that important clues about the occurrence of tippings points can be revealed from different sensitivity analysis methodologies, if proper statistical and graphical measures are used. The results raise awareness about how tipping points affect temporal model responses in ecological simulation models, and may also be more generally applicable for nondeterministic models that cannot be analysed using bifurcation analysis.
\end{abstract}

Keywords and phrases: Allee effect, bifurcation analysis, sensitivity analysis, sampling method, tipping point

Mathematics Subject Classification: 49Q12, 34K18, 65C05

\section{Introduction}

Ecological researchers and managers of natural systems commonly use simulation models to understand and predict the effects of drivers on ecological systems [29]. Most ecological systems are Complex Adaptive Systems with many interacting, biotic components, and feedbacks [30]. The applicability of simulation models is determined not only by the validity of these models (i.e. whether the models are a proper and useful simplified representation of the modelled system), but also by our ability to analyse them. Model analysis is vital during all steps in the development and use of ecological models [15,29], including model testing, calibration, validation, uncertainty estimation, and gaining a better understanding of

\footnotetext{
${ }^{*}$ Corresponding author. E-mail: guus.tenbroeke@wur.nl
} 
model behaviour. Reflecting the complexity of the modelled system, many simulation models are also of considerable complexity. This generates a demand for the development and application of model analysis methodologies. Without the right methodologies a proper model analysis is not possible, and simulation models are of limited use.

We consider two important tools for model analysis, namely sensitivity analysis and bifurcation analysis. Sensitivity analysis quantifies the effects of changes in the parameters (including initial conditions) on the model output $[4,5,14,24]$. Its main use is to estimate which parameters are the most influential on certain model outputs. This information is useful for instance to quantify the information content of data for a given model, or to establish what parameters to focus on in validation experiments. Bifurcation analysis, on the other hand, considers changes in the topology of the phase space (i.e. qualitative changes) of the model with changes in parameters (including initial conditions). A lack of change in the topology with parameter changes may indicate resilience, i.e. the capacity of the modelled system to withstand pressures without undergoing drastic changes [40]. Changes in topology may indicate tipping points that can lead to catastrophic shifts - fast, large-scale, and irreversible changes from one system state to another under relatively small changes in drivers $[3,11,27,28]$. Tipping points are either bifurcations or separatrices $[13,18,31,41]$. A bifurcation is a specific parameter setting at which a qualitative change in model behaviour occurs. A separatrix is a manifold in state space that separates two domains of attraction of neighbouring attractors. In the latter case there are at least two alternative types of asymptotic model behaviour, and the initial conditions determine to which attractor the model will eventually evolve if no further manipulations of the system occur.

For many ecological management applications there is an interest in tipping points [11] and resilience, i.e. the asymptotic model behavioural features. For those applications bifurcation analysis would be the preferred method for analysing models. However, the application of bifurcation analysis is restricted by the complexity, as well as the type of many ecological models. Bifurcation analysis is well-suited for the analysis of deterministic dynamic models (DAEs or differential and algebraic equations) with a limited number of model variables and parameters, for which semi-automated tools are available, such as MATCONT [7] and AUto07P [8]. For complex models with a large number of parameters it is often not feasible to analyse the model that way. Furthermore, many types of models cannot at all be analysed using bifurcation analysis. The reason is that for differential equation (ODE) models, bifurcation analysis methods rely on the use of derivatives of the model equations, but these are not available for models with stochastic terms, or models where we have no explicit model equations available, such as agent-based models. Features like tipping points and resilience can nevertheless be expected in complex ecological simulation models. Therefore there is a dire need for methodologies that can analyse these models $([6,10,19])$.

Methodologies of sensitivity analysis may be useful as an alternative for analysing more complex and nondeterministic ecological models. Sensitivity analysis methodologies are broadly categorised as being either local or global [5]. Local methodologies quantify the sensitivity at a specific point in parameter space, whereas global quantify it across a range of parameter space. In addition, there are hybrid methodologies $[22,33]$ that apply local methodologies at different points in parameter space to obtain a distribution of local sensitivity indices across a range of parameter space. Local methodologies linearise the output around the point at which they are applied to reveal detailed information limited to the neighbourhood of this point, omitting nonlocal interaction effects. This linearisation involves the determination of partial derivatives and thus meets the same limitation as bifurcation analysis. Global methodologies, on the other hand, reveal information that is valid over the full parameter range under investigation, by sampling from this range, and thus include nonlocal interaction effects. Model output is typically aggregated into statistical measures across the sample range, which results in the loss of more detailed information about model behaviour, including resilience and tipping points. The issue is demonstrated by Figure 1, which contains the bifurcation diagram of the one-dimensional Allee model, 


$$
\frac{d Y}{d t}=Y(Y-\zeta)(\kappa-Y)-H Y
$$

where $Y$ is the population density, $\kappa$ the carrying capacity, $\zeta$ the Allee threshold, and $H$ a harvest rate constant. In the bifurcation diagram $\kappa=1, \zeta=0.2$, and $H$ is varied. For $H>0.16$ the population goes extinct for all initial conditions, whereas for $H<0.16$ there are two alternative attractors, namely a stable positive steady state and the stable zero state, each with their own domain of attraction. Global sensitivity analysis methodologies typically aggregate samples (output) from the two domains of attraction into the same statistics. As a result, the qualitative difference in model behaviour between the domains of attraction is obscured. The analysis may correctly show that $H$, on average, is an influential parameter, but it will not show that $H$ is crucial in determining whether the model evolves to a positive stable state or that a tipping point exists.

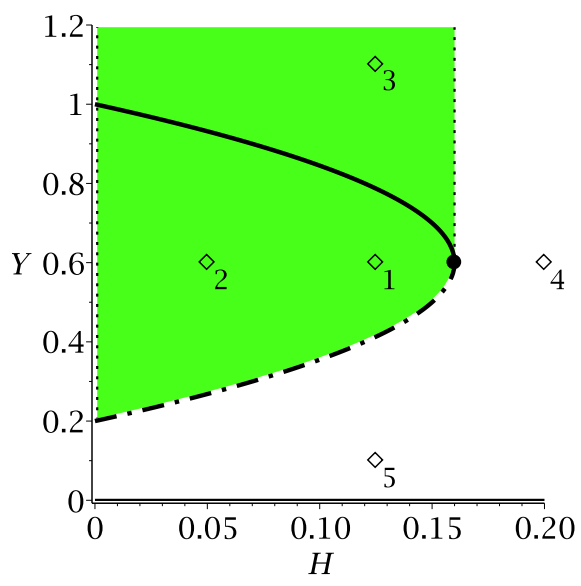

FiguRE 1. Bifurcation diagram of a model with tipping points Eqn. (1.1), with $\kappa=1$ and $\zeta=0.2$. A bifurcation occurs at $H=0.16$. For $H<0.16$ there is bistability. Initial conditions $Y(0)$ in the green domain of attraction (samples 1, 2, and 3) evolve to the positive stable attractor indicated by the solid curve. Initial conditions in the white domain of attraction (samples 4 and 5) evolve to extinction. Statistics that are generated without distinction between these domains of attraction will be poor on information that is useful for tipping point analysis.

As it is, there are no standardised sensitivity analysis methodologies that are particularly suited to reveal or deal with tipping points. Ten Broeke et al. [36] concluded that a one-factor-at-a-time approach is the best methodology for locating tipping points in ABMs. It is however a brute force method (i.e. based on performing many simulation runs), and hence it has obvious limitations in terms of computational costs. Because of the nondeterministic (i.e. stochastic) nature of many complex ecological models (including ABMs) many repetitive simulation runs have to be performed at different values of a single parameter before a distinction can be made between effects of tipping points and those of model stochasticity. This limits the application value of such sensitivity analysis methodologies for locating tipping points.

In this paper, we deal with the question of whether methodologies for sensitivity analysis can have a practical use for studying asymptotic features like tipping points and resilience in ecological models. By means of an example for which all the asymptotic features can be analysed using bifurcation analysis, we show how much information on tipping points and resilience can be obtained using methodologies for 
TABLE 1. List of all general symbols used in the paper.

\begin{tabular}{ll}
\hline Symbol & Meaning \\
\hline $\mathbf{A}$ & Sampling matrix (used only in Appendix) \\
$\mathbf{A}_{B}$ & Recombinant sampling matrix (used only in Appendix) \\
$\mathbf{B}$ & Alternative sampling matrix (used only in Appendix) \\
$\mathbf{B}_{A}$ & Recombinant sampling matrix (used only in Appendix) \\
$E(\cdot)$ & Expected value \\
$e_{i, j}$ & Elasticity \\
$f$ & Function; model output \\
$G$ & Curve corresponding to global bifurcation \\
$g(Y, \theta, t)$ & Vector field of oDE model (used only in Appendix) \\
$H$ & Curve corresponding to Hopf bifurcation \\
$i$ & Index for model parameters \\
$\mathbf{J}$ & Jacobian matrix \\
$I^{N_{p}}$ & N - dimensional unit hypercube (used only in Appendix) \\
$j$ & Index for model outputs \\
$l$ & Index for model parameters \\
$m$ & Index for model outputs \\
$N_{p}$ & Total number of parameters \\
$N_{s}$ & Sample size in sampling method by [26] \\
$N_{v}$ & Total number of outputs \\
$n$ & Number of samples per parameter in the factorial design \\
$P$ & Probability density function (used only in Appendix) \\
$S_{i, j}$ & Global first order variance-based sensitivity \\
$\tilde{S}_{i, j}$ & Global total effect variance-based sensitivity \\
$s_{i, j}$ & Local sensitivity \\
$\tilde{s}_{i, j}$ & DELSA local sensitivity \\
$t$ & Time \\
$U(\cdot)$ & Uniform probability density \\
$V(\cdot)$ & Variance \\
$V_{i}$ & Partial variance attributed to parameter $i$ \\
$W^{s}$ & Stable manifold in state space \\
$W^{u}$ & Unstable manifold in state space \\
$Y$ & Model output vector with elements $Y_{j}$ \\
$\Theta$ & All parameters $\theta_{i}$ \\
$\theta_{i}$ & Model parameter or initial condition \\
\hline &
\end{tabular}

sensitivity analysis. The paper is organised as follows. In Section 2 three (groups of) methodologies for performing sensitivity analysis are discussed. In Section 3 the well-known Bazykin-Berezovskaya predatorprey model is introduced as a case study. The results of the analysis of the test case using sensitivity analysis methodologies are given in Section 4. Section 5 contains the discussion and conclusions.

\section{Sensitivity analysis methodologies}

In this section we provide a general overview of the sensitivity analysis methodologies that are applied in this paper. This section can be skipped without loss of readability by readers who have extensive knowledge of methodologies for sensitivity analysis, or who wish to skip the technical details.

\subsection{Local sensitivity analysis}

Local parameter sensitivities are typically expressed as the partial derivative of the model output with respect to a selected parameter (including initial conditions) 


$$
s_{i, j}(t)=\frac{\partial Y_{j}(t)}{\partial \theta_{i}}
$$

where $t$ is time (i.e. these sensitivities may vary over time), $Y_{j}$ are the output variables, $\theta_{i}$ the parameters (including initial conditions), $i=1,2, \ldots, N_{p}$ with $N_{p}$ the number of parameters, and $j=1,2, \ldots, N_{v}$ with $N_{v}$ the number of variables or model outputs (see Table 1 for an overview of the used symbols). Eqn. (2.1) may be estimated for ODE models using the direct differential method [9] (Appendix A). Sensitivity indices are expressed in this method as additional differential equations to be solved alongside the original ODE model. Alternatively, finite differences methods can be used to estimate Eqn. (2.1) based on e.g. the difference between a model run in the nominal point and a model run with a slightly different value for the parameter for which the sensitivity is estimated.

In a one-at-a-time sensitivity analysis the local partial derivatives of model outputs with respect to any number of (preferably all) parameters are determined around a single point. Local sensitivity indices Eqn. (2.1) cannot be directly compared because parameters may have different units. Therefore, in addition to local sensitivity indices one usually reports elasticities, which have been normalised using the nominal parameter values

$$
e_{i, j}(t)=\frac{\theta_{i}}{Y_{j}(t)} \frac{\partial Y_{j}(t)}{\partial \theta_{i}}
$$

Elasticities have a straightforward interpretation. For example, an elasticity of 5 indicates that a $1 \%$ change in the parameter causes a $5 \%$ change in the output with respect to the nominal point. Elasticities are not well-defined when the output variable $Y_{j}$ goes to zero. For negative parameter values, a positive elasticity would indicate that a positive parameter change causes a negative change in the output. It is therefore common to report the absolute value of the elasticity, which expresses the magnitude of the output change that is caused by a parameter change.

Local sensitivity analysis is a useful methodology for assessing which parameters in ecological models are the most influential around a nominal point. It has the added advantage of being computationally cheap. Since local sensitivity analysis involves linearisation of the output response around the nominal point, it omits nonlocal interaction effects. Some care should be taken when applying local sensitivity analysis to models with tipping points, because at a tipping point there is a discontinuity in the output and the partial derivatives are thus not well-defined.

Besides local sensitivity analysis methodologies based on partial derivatives, one can also perform onefactor-at-a-time (OFAT) sensitivity analysis. Similar to other local sensitivity analysis methodologies, OFAT considers only changes in a single parameter with respect to a nominal point, while keeping all other parameters constant. Instead of linearising the output around the nominal point, in OFAT the parameter is varied stepwise over a larger range. The model output is plotted as a function of the varied parameter. OFAT is not directly aimed at quantifying sensitivities, but is useful for revealing qualitative relations between individual parameters and the model output. OFAT can reveal whether the output changes linearly or non-linearly as a function of changes in a single model parameter, and thus can help to reveal whether tipping points are crossed as the parameter is changed [36].

\subsection{Global sensitivity analysis}

Global sensitivity analysis considers parameter changes over a larger range of parameter space and may thus include nonlocal interaction effects. The range is defined by assigning uniform probability density functions to the parameters that are included in the analysis. The variation in the output variables over the range is then attributed to the variations in the different parameters. The Sobol' method [32] is one of the most commonly used methodologies for global sensitivity analysis. This method is based on a decomposition of the output variance under the assumption that all parameters are independent $[16,26]$ 


$$
V\left(Y_{j}(t)\right)=\sum_{i} V_{i}(t)+\sum_{l>i} V_{i, l}(t)+\ldots+V_{1,2, \ldots, N_{p}}(t)
$$

where $V\left(Y_{j}(t)\right)$ is the total output variance over the considered region of parameter space at time $t$, $V_{i}(t)$ is the part of the variance that is attributed to the parameter $\theta_{i}$, and $V_{i, l}(t)$ is the part that is attributed to the interaction between $\theta_{i}$ and $\theta_{l}$. Higher order terms represent higher order interaction effects. Sensitivity indices are defined by normalising the terms of Eqn. (2.3) through division by the total variance. The most commonly reported sensitivity indices are the first-order index and the total-order index [25]. The first-order index estimates the variance that is explained by a single parameter excluding interaction effects,

$$
S_{i, j}(t)=\frac{V_{i}(t)}{V\left(Y_{j}(t)\right)} .
$$

The total-order sensitivity includes all the interaction effects with other parameters,

$$
\tilde{S}_{i, j}(t)=\frac{1}{V\left(Y_{j}(t)\right)}\left(V_{i}(t)+\sum_{l} V_{i, l}(t)+\ldots+V_{1,2, \ldots, N_{p}}(t)\right) .
$$

First- and total-order indices always attain values between 0 and 1 . However, the sum of the total-order indices exceeds 1 if the model has interaction effects. A large difference between Eqn. (2.4) and Eqn. (2.5) indicates that interaction effects are influential.

The first-order sensitivity Eqn. (2.4) can be expressed in terms of conditional variances and expectations of the model output [32] (Appendix D)

$$
S_{i, j}(t)=\frac{V_{\theta_{i}}\left(E_{\theta_{\sim i}}\left(Y_{j}(t) \mid \theta_{i}\right)\right)}{V\left(Y_{j}(t)\right)},
$$

where $E(\cdot)$ is the expectation value, $V(\cdot)$ the variance (see also Table 1 ), and $\sim$ indicates 'all except', i.e. all parameters are varied except parameter $\theta_{i}$.

To evaluate Eqn. (2.6), we first compute the expectation value of the model output over all other parameters, keeping $\theta_{i}$ fixed. We then compute the variance of the resulting expectation value over the possible values of $\theta_{i}$. Similarly, the total-order sensitivity indices may be expressed as

$$
\tilde{S}_{i, j}(t)=\frac{E_{\theta_{\sim i}}\left(V_{\theta_{i}}\left(Y_{j}(t) \mid \theta_{\sim i}\right)\right)}{V\left(Y_{j}(t)\right)}=1-\frac{V_{\theta_{\sim i}}\left(E_{\theta_{i}}\left(Y_{j}(t) \mid \theta_{\sim i}\right)\right)}{V\left(Y_{j}(t)\right)},
$$

where the law of total variance is used to obtain the rightmost expression.

If for ODE models explicit expressions for the steady states are available, Eqns. (2.6-2.7) are evaluated analytically by inserting the steady state values to which the model evolves given the choice of parameters and initial conditions, and performing integrations over the parameters to calculate the means and variances. If explicit expressions are not available, a number of methodologies are available to estimate Eqns. (2.6-2.7) based on samples from the parameter space (i.e. brute force approach) [26]. The most direct sampling method is a factorial design, in which the considered parameter space is divided into (possibly equidistant) steps, obtaining a chessboard-like grid. The intersection points of this grid are used as sample points. A grid of five points for four parameters thus gives $5^{4}=625$ possible parameter combinations and the same number of model simulations (excluding replicates to account for stochasticity). 
The factorial design provides a straightforward way to evaluate Eqns. (2.6-2.7), but since the number of sample points increases exponentially with the number of parameters, it is impractical for models with a large number of parameters. For models with many parameters, more cost efficient sampling methodologies have been proposed. One such method is aimed at the estimation of the partial variances based on covariances [26] (explained in detail in Appendix F). This method has limitations in that it can yield negative estimates for the partial variances. In this paper we will use both the factorial design and the method by [26] to estimate global sensitivities. Note that the Sobol' method in general is not applicable if the parameters are dependent, because the variance decomposition Eqn. (2.3) holds only for independent parameters.

\subsection{Hybrid sensitivity analysis}

Hybrid methodologies of sensitivity analysis compute local sensitivity indices at various points in parameter space, thus combining local and global sensitivity analysis. The local sensitivity index in each point measures the local sensitivity around that point. The distribution of sensitivity indices across a range of parameter space measures the global sensitivity over the region. The DELSA methodology [22] converts local sensitivity indices into variance-based sensitivity indices, enabling direct comparison with the Sobol' methodology. Computing the variance of a Taylor expansion of the model output around the point where the local sensitivity index is evaluated yields, [21,22]

$$
V\left(Y_{j}(t)\right) \approx \sum_{i=1}^{N_{p}}\left|\frac{\partial Y_{j}(t)}{\partial \theta_{i}}\right|_{\Theta}^{2} V\left(\theta_{i}\right)
$$

where the summation runs over all parameters, $\Theta=\left(\theta_{1}, \theta_{2}, \cdots, \theta_{N_{p}}\right) \in \mathbb{R}^{N_{p}}$ denotes the point in parameter space where the local sensitivity index is evaluated. $V\left(\theta_{i}\right)$ is the variance of the parameters around this point, given the (uniform) probability distribution that is assigned to the parameters. Each term in the sum denotes the output variance that is attributed to the corresponding parameter. Sensitivity indices are obtained by normalising these terms with respect to the total output variance around the points

$$
\tilde{s}_{i, j}=\frac{1}{V\left(Y_{j}(t)\right)}\left|\frac{\partial Y_{j}(t)}{\partial \theta_{i}}\right|_{\Theta}^{2} V\left(\theta_{i}\right), \quad i=1,2, \cdots, N_{p} .
$$

Note that this sensitivity index $\tilde{s}_{i, j}$ is a local sensitivity measure that estimates the sensitivity around the point where Eqn. (2.9) is evaluated. Information on global sensitivities is obtained by considering the probability density of Eqn. (2.9) across a range of parameter space. The DELSA methodology has the advantage of measuring not only global sensitivities over such a range, but also giving more detailed results for points within this range. For example, a parameter may be shown to be influential in certain parts of parameter space, but not in other parts. Unlike the Sobol' method, which is properly normalised only for independent parameters, DELSA does not assume that the model parameters are independent. In addition, the computational costs have been reported to be lower than the costs of the Sobol' method [22], but nevertheless are still much higher than the costs of local sensitivity analysis.

\section{Case description}

\subsection{Model}

Our case study involves the analysis of the Bazykin-Berezovskaya predator-prey model with an Allee effect for the prey species [2]. The Allee effect refers to the observation that many populations of species do not only suffer from detrimental effects when densities are high, i.e. because of intraspecific competition, but also when densities are low $[1,17]$. This may result, for instance, from difficulties in finding mates 
TABLE 2. List of the variables, parameters and initial conditions used in the Allee model. Also indicated are the nominal values (if applicable) and whether they are fixed or not in the sensitivity analysis.

\begin{tabular}{llll}
\hline Symbol & Nominal value & Fixed/Free & Meaning \\
\hline$Y_{1}$ & - & - & Prey population density \\
$Y_{2}$ & - & - & Predator population density \\
\hline$Y_{1}(0)$ & 0.9 & Fixed & Initial condition of $Y_{1}$ \\
$Y_{2}(0)$ & 0.1 & Free & Initial condition of $Y_{2}$ \\
$\gamma$ & 1. & Fixed & Conversion factor \\
$\kappa$ & 1. & Fixed & Prey carrying capacity \\
$h$ & 0.9 & Free & Predator mortality rate \\
$\zeta$ & 0.5 & Free & Allee threshold (prey) density \\
\hline$H$ & - & - & Prey harvesting rate (Eqn. 1.1) \\
\hline
\end{tabular}

or cooperative feeding [17], or a positive relationship between a component of individual fitness and the number of conspecifics [34]. A distinction is commonly made between weak and strong Allee effects [35]. A weak Allee effect indicates there are negative effects but not such that the population will go extinct. A strong Allee effect, as occurs in the Bazykin-Berezovskaya model, indicates there is a certain threshold density below which the population will go extinct.

The Bazykin-Berezovskaya model is well-known and mathematically tractable, and has been extensively analysed. The model is well-suited to demonstrate the issues we address in this paper, because all relevant types of tipping points occur in the model, namely separatrices (and hence alternative attractors), local bifurcations, and also global bifurcations that involve the (dis)appearance of separatrices. This makes it an ideal test case to study what information about tipping points can be found based on the application of methodologies for sensitivity analysis.

The nondimensional Bazykin-Berezovskaya model reads

$$
\begin{aligned}
\frac{d Y_{1}}{d t} & =Y_{1}\left(Y_{1}-\zeta\right)\left(\kappa-Y_{1}\right)-Y_{1} Y_{2}, \\
\frac{d Y_{2}}{d t} & =\gamma\left(Y_{1}-h\right) Y_{2},
\end{aligned}
$$

where $Y_{1}$ is the prey density, $Y_{2}$ the predator density, $\kappa$ the carrying capacity (the positive monoculture steady state density of the prey species), $\gamma$ the conversion factor from prey to predator, and $h$ the predator mortality rate, scaled to the conversion factor $\gamma$. Parameter $\zeta$ represents the Allee threshold. A concise overview of the model variables and parameters is given in Table 2 .

\subsection{Bifurcation analysis}

The Bazykin-Berezovskaya model has been analysed thoroughly in the literature. In particular numerical techniques based on defining boundary value problems have been used to localize a heteroclinic pointto-point connection [38]. This connection is not structurally stable, and has a biological interpretation as overexploitation. We summarise these results for convenience before proceeding to the sensitivity analysis.

Eqn. (3.1) has four steady state solutions, three trivial ones $\left(Y^{0}=[0,0], Y^{z}=[\zeta, 0], Y^{k}=[\kappa, 0]\right)$, and a nontrivial one

$$
Y^{*}=\left[h, h \zeta-\kappa \zeta-h^{2}+h \kappa\right] .
$$

The Jacobian matrix for the nontrivial steady state is 


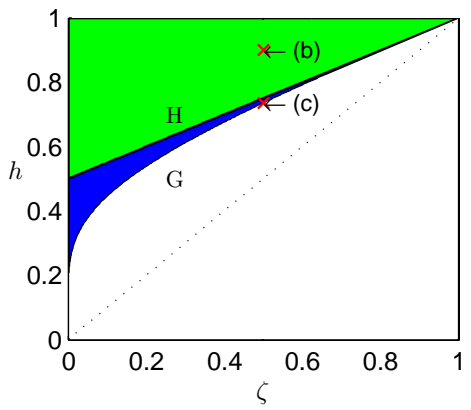

(a)

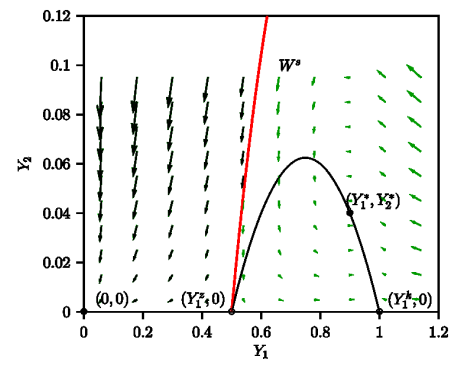

(b)

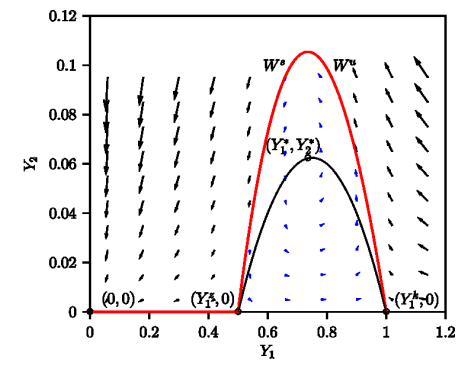

(c)

Figure 2. (a): Two-parameter bifurcation diagram of the Bazykin-Berezovskaya model for $\zeta$ and $h$, with other parameters at their nominal values. $H$ indicates the Hopf bifurcation curve. $G$ indicates the global bifurcation curve. In the green region there are two alternative attractors, namely $Y^{*}($ Eqn. 3.2$)$ and $Y^{0}=[0,0]$. In the blue region the nontrivial positive steady state has turned into a periodic attractor. In the white region the community always goes extinct. The dotted line indicates a transcritical bifurcation curve. (b): The vector field for $Y_{1}$ and $Y_{2}$ with all parameters in the nominal point (marked as (b) in Fig. 2a) shows that there is bistability. The black line is the nullcline where the time-derivative of $Y_{1}$ is zero. The red line $W^{s}$ is the stable manifold that terminates at $\left(Y_{1}^{z}, 0\right)$ and acts as a separatrix. (c): At $h \approx 0.735442$ and all other parameters at their nominal value (marked as (c) in Fig. 2a), the stable manifold $W^{s}$ and the unstable manifold $W^{u}$ connect the steady states $\left(Y_{1}^{z}, 0\right)$ and $\left(Y_{1}^{k}, 0\right)$ and act as a separatrix. Inside the manifold, the solution is a stable limit cycle, whereas outside the manifold the solution goes to extinction.

$$
\left.\mathbf{J}\right|_{Y=Y^{*}}=\left(\begin{array}{cc}
h(\zeta+\kappa-2 h) & -h \\
\gamma(h-\kappa)(\zeta-h) & 0
\end{array}\right) .
$$

The determinant of $\mathbf{J}$ is used to determine the transcritical bifurcation of the nontrivial steady state, i.e. the point where the predator species can enter the system and sustain itself. The trace is used to determine the Hopf bifurcation, i.e. the point where the nontrivial stable steady state becomes unstable and periodic behaviour appears, giving rise to (in this case stable) limit cycles.

The bifurcation diagram displayed in Figure 2a shows the asymptotic behaviour of the model as function of $\zeta$ and $h(\gamma=1, \kappa=1)$. Three transcritical bifurcations are found at $h^{0}=0, h^{z}=\zeta$, and $h^{k}=\kappa$, and the Hopf bifurcation occurs at

$$
h^{H}=\frac{1}{2}(\kappa+\zeta),
$$

which is indicated by the curve $H$ in Figure 2a. The model displays bistability for a significant part of parameter space. When the nontrivial steady state is positive, there exists a unique manifold that terminates at the trivial steady state $Y^{z}$ and acts as a separatrix (Figure 2b). Initial conditions on the right hand side of the separatrix converge to $Y^{*}$ and on the left hand side to the trivial steady state $Y^{0}$. Thus, in the green parameter region in Figure 2a the model may converge to the nontrivial steady state $Y^{*}$, or to the trivial steady state $Y^{0}$, depending on the initial conditions. In the blue parameter region the model may converge either to the limit cycle around $Y^{*}$, or to $Y^{0}$.

The heteroclinic point-to-point connection links the saddle steady state $Y^{k}$ to the saddle steady state $Y^{z}$, as the stable manifold $W_{s}$ belonging to $Y^{z}$ overlaps with the unstable manifold $W_{u}$ belonging to 
$Y^{k}$ (see Figure $2 \mathrm{c}$ for the connection at the parameter set $\zeta=0.5, h^{G} \approx 0.735442$ ). The two-parameter continuation of this curve, indicated by $G$ in Figure 2a, is obtained by using AUTO[8] with a set of boundary conditions [38,39]. For values of $h>h^{G}$ (green and blue parameter regions) the system displays bistability. For values $h<h^{G}$ the separatrix has disappeared, and $Y^{0}$ is the sole attractor (white parameter region).

\section{Results of sensitivity analysis}

In this section we apply the sensitivity analysis methodologies that are presented in Section 2 to the Bazykin-Berezovskaya model and discuss the obtained results. We consider only the predator density $Y_{2}$ as model output and parameters $h$ and $\zeta$ as inputs to demonstrate the principles. The parameters $\gamma$ and $\kappa$ are fixed at the nominal values, and $Y_{1}(0)$ is fixed such that its value lies between $\kappa=1$ and the highest sampled value of $\zeta$. Note, that for the sensitivity analysis we assume a researcher who is aware that tipping points may exist, but who is not able for whatever reason to perform bifurcation analysis. The aim is to evaluate which of the sensitivity analysis methodologies can be useful in detecting, or revealing clues about, tipping points such as the transcritical bifurcation, the Hopf bifurcation, the separatrix and the heteroclinic connection.

\subsection{Results of local sensitivity analysis}

A classical starting point of a sensitivity analysis is to quantify the local sensitivities Eqn. (2.1) as given in subsection 2.1. For finite values of $t$ we use the direct differential method (Appendix A) to determine these local sensitivities (details of the calculation are given in Appendix B). The results show that the predator density initially is most sensitive to the initial condition $Y_{2}(0)$, which is an obvious result (Figure 3). As the simulation proceeds, the sensitivity to $Y_{2}(0)$ decreases to zero, while the sensitivity to $h$ becomes the largest. The elasticities show the same outcome: the sensitivity of the model for $Y_{2}(0)$ decreases to zero, while the sensitivity for $h$ becomes the largest.

For long simulation times (effectively $t \rightarrow \infty$ ) the model and its parameter sensitivities evolve to steady state values. The steady state sensitivities can also be determined analytically by taking the derivative in the positive steady state $Y^{*}$ Eqn. (3.2),

$$
\begin{gathered}
s_{\zeta}^{*}=\frac{\partial Y_{2}^{*}}{\partial \zeta}=h-\kappa, \\
s_{h}^{*}=\frac{\partial Y_{2}^{*}}{\partial h}=\kappa+\zeta-2 h,
\end{gathered}
$$

and

$$
s_{Y_{2}(0)}^{*}=0,
$$

where the index $j$ in Eqn. (2.1) is dropped from the notation, and for the index $i$ we write $\zeta, h$, or $Y_{2}(0)$. Table 3 gives these steady state sensitivities, along with the associated elasticities Eqn. (2.2). The outcomes show a close match with those in Figure 3 for sufficiently large values of $t$.

Note that the above results estimate the effects of small parameter changes around the nominal point. For larger parameter changes with respect to the nominal set, tipping points and other nonlinearities may become important, in which case these results are no longer valid. For example, whereas $s_{Y_{2}(0)}=0$ in the nominal setting, Figure $2 \mathrm{~b}$ shows that for larger parameter changes the value of $Y_{2}(0)$ determines whether the model evolves to the positive steady state, or to extinction. Thus, anywhere away from the separatrix $W^{s}$ the sensitivity $s_{Y_{2}(0)}$ asymptotically goes to zero, but at the separatrix the sensitivity is not well-defined. 


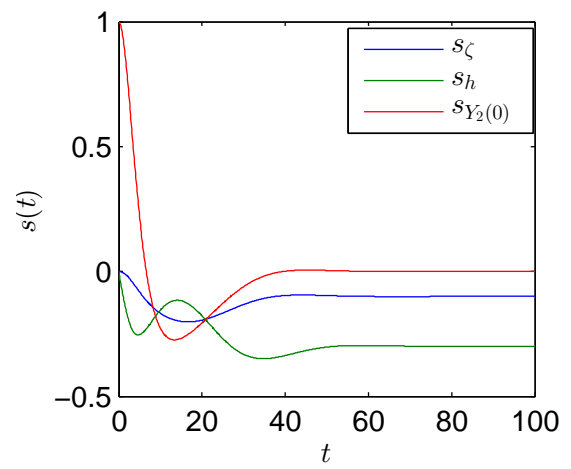

(a)

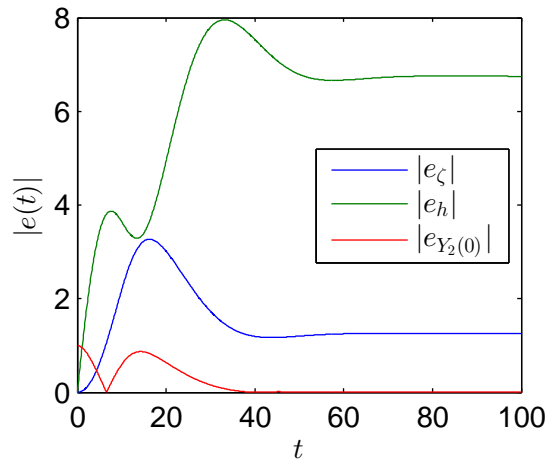

(b)

Figure 3. (a) : The local sensitivities of $Y_{2}(t)$ of the Bazykin-Berezovskaya model with respect to parameters $\zeta$ (in blue), $h$ (in green), and the initial condition $Y_{2}(0)$ (in red). Other parameters are set at their nominal values. Over time the sensitivity to $Y_{2}(0)$ decreases to around zero, while that of $h$ seems to be the largest. (b): The respective elasticities, i.e. dimensionless sensitivities. Also in this case the sensitivity to $h$ is found to be the largest.

TABLE 3. Local and global sensitivities in the steady state $\left(Y_{1}^{*}, Y_{2}^{*}\right)$ for the output variable $Y_{2}$. The local sensitivities and elasticities are computed at the nominal point (Table 2). The first-and total-order global sensitivities Eqns. (2.6-2.7) are computed over the stable steady state region (green) in Figure 2a, assuming that the initial conditions are such that the model converges to the positive steady state $Y^{*}$. The computation of these global sensitivity indices is given in Appendix E. We have dropped the index $j$ in Eqn. 2.1 from the notation.

\begin{tabular}{llll}
\hline & $\zeta$ & $h$ & Description \\
\hline$s_{i}^{*}$ & -0.1 & -0.3 & Local sensitivity Eqn. (2.1) in nominal point \\
$\left|e_{i}^{*}\right|$ & 1.25 & 6.75 & Local elasticity Eqn. (2.2) in nominal point \\
\hline$S_{i}^{*}$ & .593 & 1.25 & First-order global sensitivity Eqn. (2.6) \\
$\tilde{S}_{i}^{*}$ & 0.267 & 0.167 & Total-order global sensitivity Eqn. (2.7)
\end{tabular}

To explore how the output responds to larger changes of individual model parameters, we perform an OFAT sensitivity analysis. The results reveal tipping points where the output goes to zero for all three parameters (Figure 4). For $h$ and $\zeta$ this indicates the presence of a transcritical bifurcation, and for $Y_{2}(0)$ it indicates the presence of a separatrix. This shows that OFAT can function as an appropriate starting point for detecting tipping points when methods of bifurcation analysis are not available. Note, however, that OFAT considers only changes in individual parameters and does not scan the full parameter space. As a result, tipping points in other parts of parameter space will not be detected.

\subsection{Results of global sensitivity analysis}

Here we apply the two methodologies to determine variance-based sensitivities Eqns. (2.6-2.7) that were introduced in subsection 2.2. The sampling of the first methodology is based on a factorial design, with $n=10,20$, and 40 equidistant values for each parameter under investigation $\left(\zeta, h\right.$ and $\left.Y_{2}(0)\right)$, giving $10^{3}=$ $1000,20^{3}=8000$ and $40^{3}=64,000$ parameter combinations, respectively. The sampling of the second methodology by [26] is based on Monte Carlo sampling, with base sample size $N_{s}=500,1000$ and 2000, giving 4000, 8000, and 16,000 parameter combinations, respectively. As both methodologies use brute force simulation, the computational costs of both methodologies can be considered to be proportional 


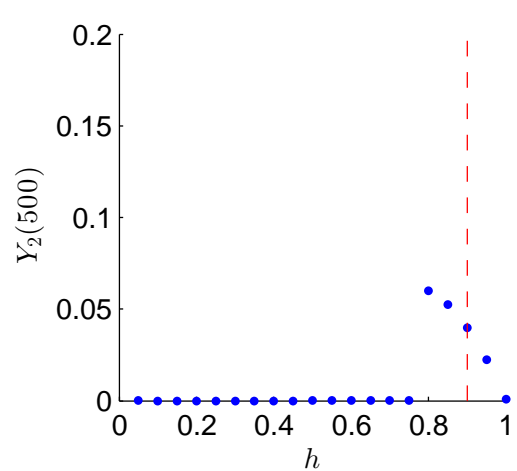

(a)

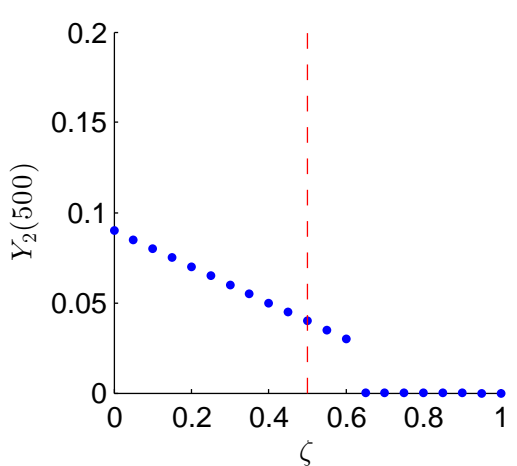

(b)

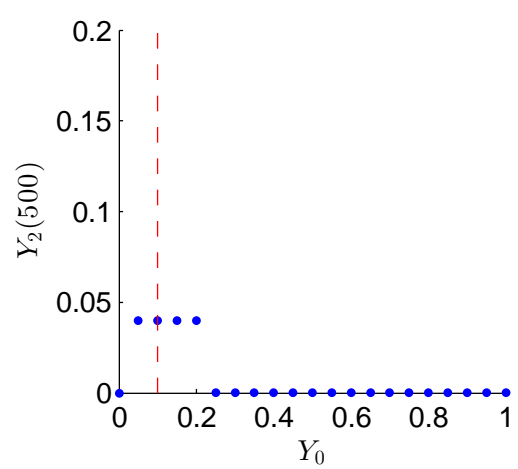

(c)

FIGURE 4. In the OFAT analysis each parameter was varied individually, starting from the nominal parameter and initial condition settings. The output is plotted as a function of the parameter, and the nominal parameter value is indicated by the red dashed line. The results at $t=500$ clearly reveal the presence of tipping points. (a): For small values of $h, Y_{2}$ converges to zero, until a tipping point is crossed where the output converges to the positive steady state. (b): For small values of $\zeta$ the model converges to the positive steady state. As $\zeta$ increases, a tipping point is crossed after which the population goes extinct. (c): For low values of $Y_{2}(0)$ the output converges to the positive steady state. Changes in $Y_{2}(0)$ do not change the value of this steady state, but as $Y_{2}(0)$ increases a separatrix is crossed after which the output goes to zero.

to the number of investigated parameter combinations. For both methodologies, $h$ and $\zeta$ were varied between 0 and 1 , and $Y_{2}(0)$ between 0 and 0.2 .

The resulting first-order Eqn. (2.4) and total-order Eqn. (2.7) global sensitivities for $Y_{2}(t=10)$, $Y_{2}(t=50)$, and $Y_{2}(t=200)$ are given in Table E.1 in Appendix E, for time points $t=10, t=50$, and $t=200$, respectively. Figure 5 shows the time evolution of the first-order and total-order sensitivities determined by using the factorial design. The sensitivities show no long-term changes after $t=100$. The fluctuations after $t=100$ are most likely caused by the fact that in some runs the system evolves to a limit cycle. The most influential parameter on shorter simulation times is $h$, whereas $\zeta$ is more influential for longer simulation times. Note that this latter result contrasts with the results of the local sensitivity analysis, which indicate that $h$ is the most influential parameter for long simulation times. We will return to this finding in section 4.3. The large differences between the first-order and total-order sensitivities indicate that interaction effects are important.

Overall there is a rather good match between the results of the factorial design and the method by [26] for $t=10$ and $t=50$. For $t=200$, however, the outcomes differ considerably. The factorial design indicates that $\zeta$ is the most influential parameter, whereas the method by [26] indicates that $h$ is the most influential. The difference between the methodologies is caused by a small number of sample points with values of $h$ close to zero. The convergence of $Y_{2}$ to the steady state value $Y^{0}$ requires a very long simulation time for small values of $h$. In the factorial design, the lowest value of $h$ in the sample grid is sufficiently large for the sample runs to have converged around $t=200$. In the method by [26], however, the sampling is random and very small values of $h$ can thus occur, at which convergence has not yet occurred around $t=200$. This explanation was checked by performing some extended simulations for these small values of $h$. After inclusion of the results of these extended simulations the method by [26] also indicates $\zeta$ to be the most influential parameter.

The aggregated statistics presented by Eqn. (2.4) and Eqn. (2.7) are not informative in terms of the detection of possible tipping points. As an alternative, non-aggregated statistics may be used, preferably 


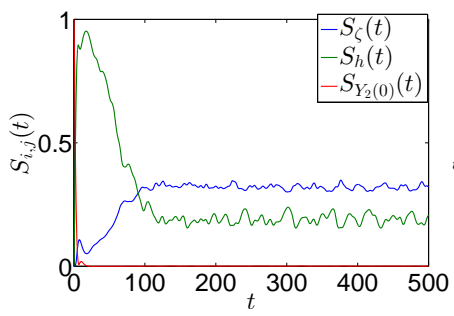

(a)

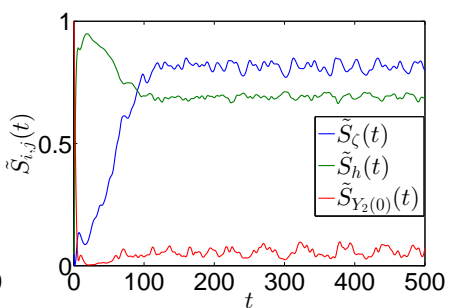

(b)

FiguRE 5. First- (a) and total-order (b) sensitivity indices as a function of time. On shorter time-scales $h$ is the most influential parameter, whereas on longer time-scales $\zeta$ is more influential. The difference between the first- and total-order indices shows that interaction effects are important. A large portion of the variance is attributed to the interaction between $h$ and $\zeta$.

in a graphical representation. Figure 6 displays the results obtained using the factorial design for $t=50$ (left panels) and $t=500$ (right panels). Samples from the three parameter regions with qualitatively different behaviour are indicated by colour and shape, where green dots indicate samples from the region with bistability where $Y^{*}$ is stable, blue diamonds are from the region with bistability where limit cycles occur, and black crosses are from the region in which there is always extinction (see also Fig. 6a). The values of $Y_{2}(t)$ are aggregated according to the parameter that is investigated while all other parameters are 'free'. For example in Figure 6c all samples are clustered per value of $\zeta$, while the other parameter values (for $h$ and $Y_{2}(0)$ ) are considered to be unknown. We refer to this as all-but-one-simultaneously (ABOS) as compared to one-factor-at-a-time (OFAT) sensitivity analysis. The red squares indicate the mean values of $Y_{2}(t)$ of the samples clustered per value of the investigated parameter. The variance of these means is the estimation of the first-order sensitivity index Eqn. (2.6). Since we are interested in revealing tipping points where the model evolves to extinction, we plot the minimum value of the limit cycle for samples that show limit cycles.

Based on the graphical ABOS results in Figure $6 \mathrm{c}$ parameter $\zeta$ can be considered to be the most influential at $t=50$. The decreasing mean (i.e. red squares) with increasing value of $\zeta$ corresponds to the decreasing green parameter region on which bistability occurs (compare to Figure 2a and Figure 6a). The cloud of black crosses is due to the fact that at $t=50$ the model still displays considerable transient behaviour. For $t=500$ the transient behaviour has disappeared and the means are near zero for $\zeta>0.5$ (Figure $6 \mathrm{~d}$ ), while also the diversity in values of $Y_{2}(t)$ decreases for increasing values of $\zeta$. It can be concluded from this Figure that the region of bistability decreases for increasing values of $\zeta$, i.e. $\zeta$ is an influential parameter in determining the bistability in the model.

In Figure $6 \mathrm{e}$ the ABOS of parameter $h$ at $t=50$ is displayed. The samples from the green parameter region are limited to $h>0.5$, and furthermore the nonzero values of $Y_{2}(t)$ increase for decreasing values of $h$. This is in accordance with the bifurcation diagram in Figure 6a. Starting from the right, the means increase then decrease, until around $h=0.5$ the means become zero. This is a strong indication of a tipping point around this value of $h$. Indeed, in Figure $6 \mathrm{~b}$ the one-parameter continuation curve of $Y^{*}$ as function of $h$ is displayed (obtained using AUTO [8]), where $\zeta=0.5$. Within the range $0.75<h<1$ the positive steady state is stable, while within $0.5<h<0.75$ it is unstable. After the destabilization at the Hopf bifurcation at $h=0.75$ the heteroclinic bifurcation occurs at $h \approx 0.735442$, and then the transcritical bifurcation at $h=0.5$. The means in Figure $6 \mathrm{f}$ seem to follow the one-parameter continuation curve of $Y^{*}$ rather faithfully. In addition, nearing the transcritical bifurcation there is a marked increase in variance. The nonzero transient behaviour is limited to very low values of $h$. This is explained by the long simulation time that is required for the system to evolve to $Y^{0}$ for small values of $h$. When $t=500$ the increase in variance is less pronounced, while the 'spike' of black crosses has disappeared. One may 
suspect the existence of bistability based on the existence of two separate clouds (namely the green and the blue ones) and the divergence between the green cloud and the red means for decreasing $h$. Although for $0.5<h<0.75$ the values of the positive samples increase with decreasing $h$, the means decrease because a larger number of samples converges to extinction.

The sensitivity of the model to the initial condition $Y_{2}(0)$ is shown in Figure $6 \mathrm{~g}$ at $t=50$ and Figure $6 \mathrm{~h}$ at $t=500$. From both figures it can be concluded that $Y_{2}(0)$ does not contribute considerably to the model output. While for $t=50$ there is still a cloud of black crosses, at $t=500$ this has disappeared. The means however do not vary considerably as function of $Y_{2}(0)$, which corresponds to the earlier findings that $Y_{2}(0)$ is not an influential parameter.

\subsection{Results of hybrid sensitivity analysis}

The DELSA methodology combines aspects of local and global sensitivity analysis and is aimed at obtaining more detailed information on the distribution of the sensitivity indices at specific point. In this case, we would like to include points from the three parameter regions in which qualitatively different behaviour occurs (see Figure 2a). For each point in the factorial design, the local sensitivity is estimated using the direct differential method (see also Appendix A). Variance-based sensitivity indices are computed around each point using Eqn. (2.9). The results are shown in Figure 7.

For the parameter region with bistability where $Y^{*}$ is stable (green), $h$ has a large peak around 1 and $\zeta$ around zero. From this it can be concluded that although in some points $\zeta$ is more influential, overall $h$ is the most influential parameter in this parameter region. This is a contrasting result compared to the results that are obtained using the Sobol' method, which indicates that $\zeta$ is overall the most influential parameter. The reason that the Sobol' method indicates $h$ as less influential is that larger values of $h$ have a negative effect on the output through lowering the steady state value (Figure 6f), but also has a positive effect because fewer model runs evolve to extinction. These two effects partly cancel each other out, resulting in a lower global sensitivity. Even though the aggregated sensitivity may be lower, $h$ is actually the most influential parameter in determining whether the steady state $Y$ is stable (Figure 6a), and it is also the most influential parameter in the steady state (Figure 7). The DELSA method allows us to discriminate between sensitivities in parameter regions with qualitatively different behaviour, and shows that indeed the ranking of sensitivities may differ between these regions. These results could not have been obtained through the Sobol' methodology, because this method assumes that the input parameters are independent. For the green region of bistability in Figure 2a, this is clearly not the case. Naive application of the Sobol' methodology to this parameter region leads to sensitivities that are not properly normalised as is shown in Appendix E. Although the DELSA method is useful for showing how sensitivities vary between regions of the parameter space, it cannot show the effects of a tipping point directly because the local sensitivities are not well-defined in a tipping point. Thus, for this example we had to assume that information on the tipping points is already known. The sampling was done such that none of the sample points were in a tipping points, where the local sensitivities are not well-defined. For long simulation times the DELSA sensitivity indices may be computed analytically by using the derivatives of the steady state value (Appendix C). 


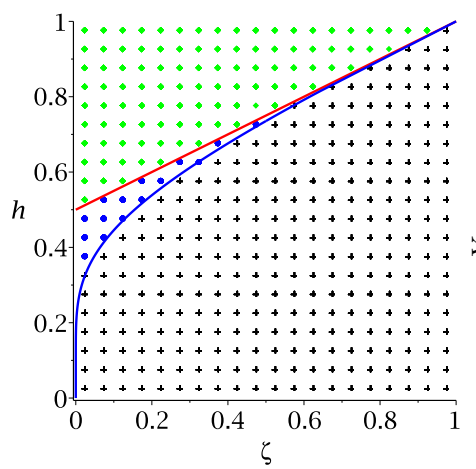

(a)

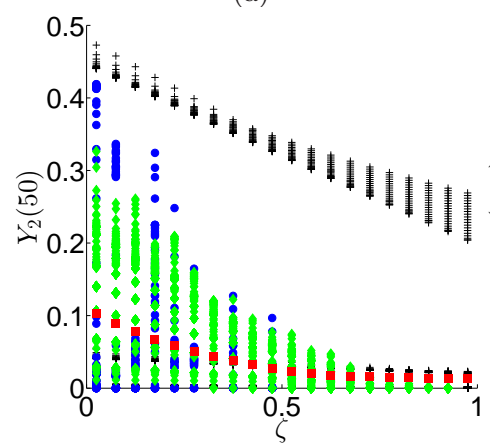

(c)

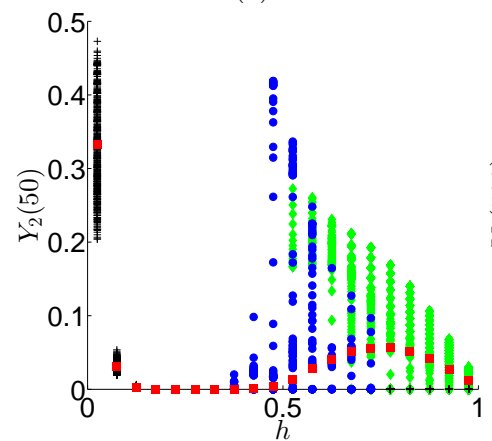

(e)

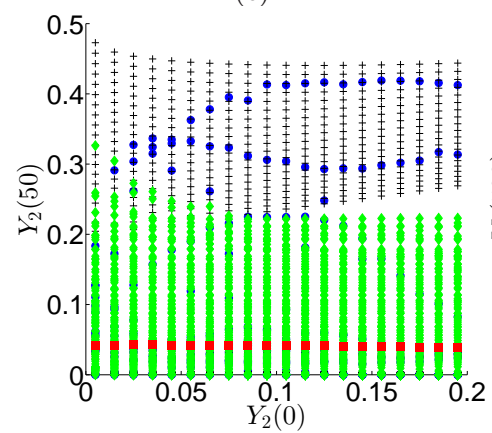

(g)

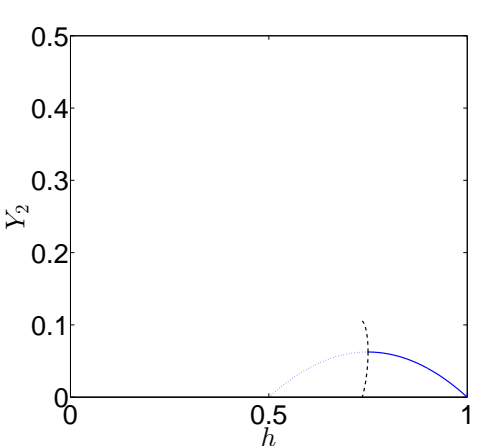

(b)

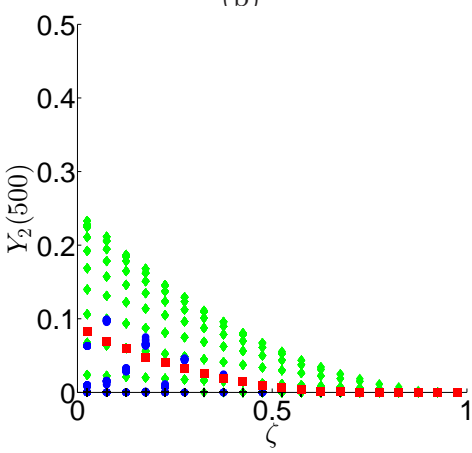

(d)

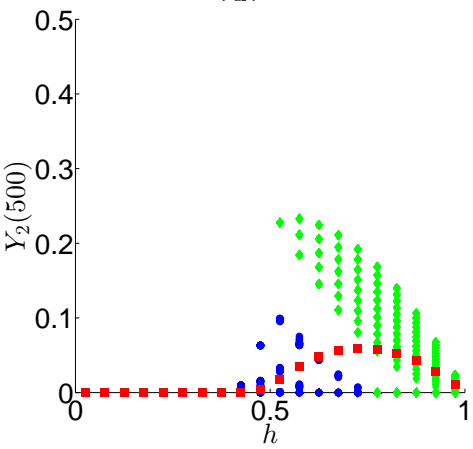

(f)

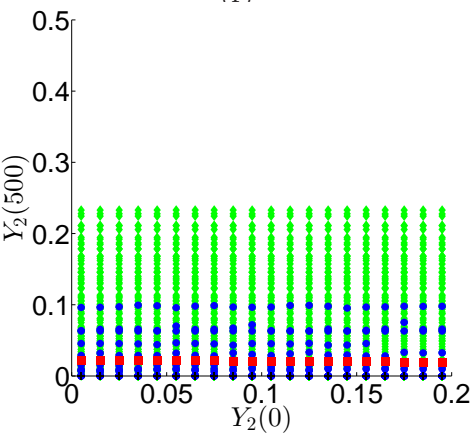

(h)

Figure 6 . ABOS results displaying $Y_{2}(50)$ and $Y_{2}(500)$ for different parameter values. A full explanation is given in section 4.2. The parameters $Y_{1}(0)=0.9875, \gamma=1$., and $\kappa=1$. are fixed, whereas $\zeta$ and $h$ are sampled at equidistant steps. The colours correspond to the regions in the bifurcation diagram Figure 2a. 


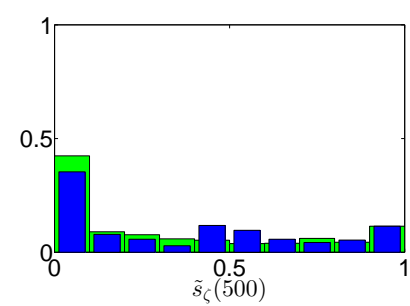

(a)

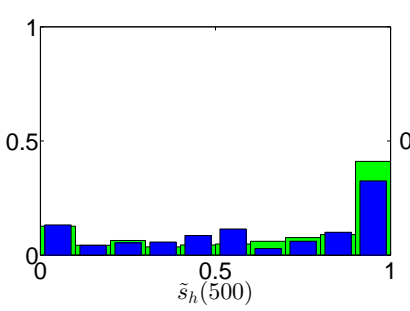

(b)

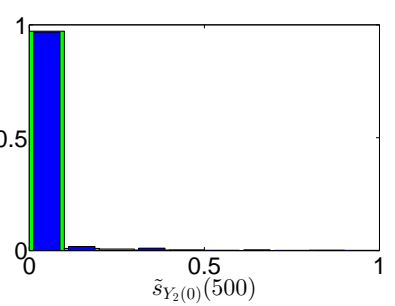

(c)

Figure 7. Histogram of DELSA sensitivity indices $\tilde{s}_{i}$ Eqn. (2.9) for $\zeta(\mathrm{a}), h(\mathrm{~b})$ and $Y_{2}(0)$ (c) at $t=500$, after all model runs have converged almost to their final behaviour. Parameters $Y_{1}(0)=0.9875, \gamma=1$., and $\kappa=1$. are fixed, whereas $\zeta, h$, and $Y_{2}(0)$ were varied using the factorial design. The different colours correspond to the regions in Figure 2a. To compare the different regions, each histogram was divided by the number of samples within the region. The results show that $h$ is overall the most influential parameter in the green region where the nontrivial steady state is stable. However, in some parts of these region $\zeta$ is more influential. In the blue region that corresponds to limit cycles, the spread in the sensitivity indices is large. 


\section{Discussion \& Conclusions}

The usefulness of simulation models for studying tipping points and resilience in ecological systems is limited by the availability of suitable methodologies for model analysis. Bifurcation analysis is the preferred methodology for detecting tipping points in deterministic models, but cannot be applied to other types of simulation models. The results in this paper show that sensitivity analysis methodologies can be useful to obtain information on tipping points in ecological models. Furthermore, it is shown that a combination of different methodologies for sensitivity analysis increases the amount of information on possible tipping points in ecological models. Below we discuss the possibilities and limitations of each of the applied methodologies in more detail.

Local sensitivity analysis is a useful methodology for assessing which parameters in ecological models are the most influential around a nominal point. This methodology considers only the linear response of the output. It is thus not well-suited for detecting tipping points. A local analysis can be extended as a one-factor-at-a-time (OFAT) analysis [36], in which the sensitivity of the model to a certain parameter is investigated by going stepwise through parameter space, where at each parameter value simulation is used to determine the model output. In effect this methodology can be considered as a discrete, brute force analogue to bifurcation analysis. Note, that since OFAT does not scan the full parameter space, it may not detect all tipping points. By including more than one parameter in the OFAT analysis the probability of detecting other tipping points (if there are any) increases. The costs of OFAT are typically not such that the inclusion of other parameters becomes prohibitively computationally expensive. In this paper, we show that OFAT can reveal separatrices and transcritical bifurcations. In [36] it was shown that OFAT can be applied to detect tipping points in non-ODE models with a comparable level of success.

Global sensitivity analysis evaluates parameter sensitivities by aggregating model output across parameter space. Time-dependent global sensitivity analysis can be used to gain insight into the dynamics of a model and to suggest parameters for more detailed investigation. In our example, time-dependent global sensitivity analysis shows that the global sensitivities continue to show oscillations on long simulation times, which indicates that some samples display limit cycles. Given that other samples evolve to a stable steady state, this indicates the existence of a Hopf bifurcation. However, standard global sensitivity analysis methodologies do not consider qualitative changes of the underlying model behaviour. As a result, these methodologies can give misleading outcomes in the presence of tipping points [37]. In our test-case the Sobol' indices indicate $\zeta$ as the most influential parameter. However, $h$ is more influential in determining whether the model converges to the positive steady state (Figures 2a and 6), and also on the value of the steady state (Figure 7). The reason that the Sobol' approach leads to a misleading conclusion is that an increase in $h$ has a positive effect on the mean output because fewer sample points converge to extinction, but also has a negative effect because value of the positive steady state is lowered. This information is revealed by applying a sampling design that allows for the calculation of (discrete) conditional means and that has sufficient coverage, e.g. the factorial design we use in this paper, combined with a proper graphical representation (Figure 6). Sparser sampling designs, such as the design in [26] have the negative side-effect that critical information about tipping points is lost. If the goal is to locate tipping points, we thus recommend to adopt a proper sampling design and data representation. Ideally such a design would scan the entire parameter space, like the factorial design, but this is often not possible in practice due to the computational expense. For larger parameter spaces, a good sampling design may retain the characteristic of the ABOs method that the model output is evaluated at a number of values for certain (combinations) of parameters, while keeping all other parameters constant. Such an approach allows to (graphically) isolate the effects of the investigated parameters as in Figure 6 and gives better insight into how these combinations of parameters affect the output, including the possible presence of tipping points. A further limitation of the Sobol' method is its reliance on the assumption that the model parameters are independent. This assumption is not always reasonable for ecological models, where certain parameter combinations may not make sense in terms of the ecological assumptions behind the model, or when we want to quantify sensitivities separately for subregions of parameter space. 
Hybrid methodologies of sensitivity analysis give a good overview of the global parameter sensitivities [22]. Furthermore, these methodologies are well-suited for the computation of parameter sensitivities for subregions within the parameter space. Thus, if the approximate location of tipping points in parameter space is known, hybrid methodologies of sensitivity analysis can be used to identify influential parameters for separate types of model behaviour, as was done in Figure 7 for the Bazykin-Berezovskaya model. A limitation of hybrid methodologies is that they do not seem to reveal information about the location of tipping points. For that task a different approach should be followed.

The results in this paper show that global methodologies of sensitivity analysis may yield misleading results when naively applied across the full parameter space of a model that contains tipping points. When methods of bifurcation analysis are applicable, these can be used to detect bifurcations before global sensitivity analysis is applied. Should tipping points be found, then we suggest to separate parameter space (and possibly state space) based on the location(s) of the tipping point(s) and perform separate sensitivity analysis for the different regions. This way the obtained results are more informative regarding the sensitivity of the model to the different inputs. For models that cannot be analysed using bifurcation analysis, sensitivity analysis methodologies can yield some information on the possible existence of tipping points. Specifically, we suggest the use of OFAT as a starting point to gain information about (the location of) tipping points. Since OFAT does not scan the full parameter space, ideally it is supplemented by a global method of sensitivity analysis to investigate interaction effects. Here we used a factorial design, which scans the full parameter space, and combined with a graphical representation as in Figure 6, can also be used gain information about tipping points. We have shown that well-known methodology can still be applied to systems with tipping points - which are in fact the most interesting ones in ecology -, provided that they are combined with a thorough insight in the bifurcation structure of the system. In line with the 'curse of dimensionality' we also admit that a complete analysis of systems with a high number of dimensions still meets with some practical limitations and can be considered an open problem. This open issue is part of ongoing research.

Acknowledgements. The research by GTB is financed by the IO/OP theme Complex Adaptive Systems at Wageningen University \& Research.

\section{Bibliography}

\section{References}

[1] W. C. Allee. Animal aggregations, a study in general sociology. The University of Chicago Press, Chicago, Ill., 1931.

[2] A. D. Bazykin. Nonlinear Dynamics of Interacting Populations. World Scientific, Singapore, 1998.

[3] C. Boettiger, N. Ross, A. Hastings. Early warning signals: the charted and uncharted territories. Theor. Ecol., 6 (2013), 255-264.

[4] F. Campolongo, A. Saltelli, J. Cariboni (2011). From screening to quantitative sensitivity analysis. A unified approach. Comput. Phys. Commun., 182 (2011), 978-988.

[5] J. Cariboni, D. Gatelli, R. Liska, A. Saltelli. The role of sensitivity analysis in ecological modelling. Ecol. Model., 203 (2007), 167-182.

[6] A. Crooks, C. Castle, M. Batty. Key challenges in agent-based modelling for geo-spatial simulation. Comput. Environ. Urban Syst., 32 (2008), 417-430.

[7] A. Dhooge, W. Govaerts, Y. A. Kuznetsov. MATCONT: a MATLAB package for numerical bifurcation analysis of ODEs. TOMS, 29 (2003), 141-164.

[8] E. J. Doedel, B. Oldeman. Auto07P: Continuation and Bifurcation software for ordinary differential equations. Concordia University, Montreal, Canada, 2009.

[9] R. P. Dickinson, R. J. Gelinas. Sensitivity analysis of ordinary differential equation systems - a direct method. Comput. Phys., 21 (1976), 123-143.

[10] T. Filatova, P. H. Verburg, D. C. Parker, C. A. Stannard. Spatial agent-based models for socio-ecological systems: challenges and prospects. Environ. Model. Softw., 45 (2013), 1-7.

[11] C. Folke, S. Carpenter, B. Walker, B., M. Scheffer, T. Elmqvist, L. Gunderson, C. S. Holling. Regime shifts, resilience, and biodiversity in ecosystem management. Annu. Rev. Ecol. Evol. Syst., 35 (2004), 557-581.

[12] G. Glen, K. Isaacs. Estimating Sobol' sensitivity indices using correlations. Environ. Model. Softw., 37 (2012), $157-166$.

[13] J. Guckenheimer, P. Holmes. Nonlinear Oscillations, Dynamical Systems and Bifurcations of Vector Fields. Springer, Berlin, 1985. 
[14] D. Hamby. A review of techniques for parameter sensitivity analysis of environmental models. Environ. Monit. Assess., 32 (1994), 135-154.

[15] A. J. Jakeman, R. A. Letcher, J. P. Norton. Ten iterative steps in development and evaluation of environmental models. Environ. Model. Softw., 21 (2006), 602-614.

[16] M. J. W. Jansen. Analysis of variance designs for model output. Comput. Phys. Commun., 117 (1999), 35-43.

[17] A. M. Kramer, B. Dennis, A. M. Liebhold, J. M. Drake. The evidence for Allee effects. Popul. Ecol., 51 (2009), $341-354$.

[18] Y. A. Kuznetsov. Elements of Applied Bifurcation Theory. Applied Mathematical Sciences 112, Springer-Verlag, New York, 2004.

[19] S. Levin, T. Xepapadeas, A. S. Crépin, J. Norberg, A. De Zeeuw, C. Folke, T. Hughes, K. Arrow, S. Barrett, G. Daily, P. Ehrlich, N. Kautsky, K. Mäler, S. Polasky, M. Troell, J. R. Vincent, B. Walker. Social-ecological systems as complex adaptive systems: modeling and policy implications. Environ. Dev. Econ., 18 (2013), 111-132.

[20] T. A. Mara, S. Tarantola. Variance-based sensitivity indices for models with dependent inputs. Reliab. Eng. Syst. Safe., 107 (2012), 115-121.

[21] A. M. Mood, F. A. Graybill, D. C. Boes. Introduction to the Theory of Statistics, McGraw-Hill, Singapore 1974.

[22] O. Rakovec, M. C. Hill, M. P. Clark, A. H. Weerts, A. J. Teuling, R. Uijlenhoet. Distributed Evaluation of Local Sensitivity Analysis (DELSA), with application to hydrologic models. Water Resour. Res., 50 (2014), $409-426$.

[23] R. Richard, J. Casas, E. McCauley. Sensitivity analysis of continuous-time models for ecological and evolutionary theories. Theor. Ecol., 8 (2015), 481-490.

[24] A. Saltelli, S. Tarantola, F. Campolongo, M. Ratto. Sensitivity Analysis in Practice. A Guide to Assessing Scientific Models. John Wiley \& Sons, 2004.

[25] A. Saltelli, M. Ratto, T. Andres, F. Campolongo, J. Cariboni, D. Gatelli, M. Saisana, S. Tarantola. Global Sensitivity Analyisis: The Primer. John Wiley \& Sons, 2008.

[26] A. Saltelli, P. Annoni, I. Azzini, F. Campolongo, M. Ratto, S. Tarantola. Variance based sensitivity analysis of model output. Design and estimator for the total sensitivity index. Comput. Phys. Commun., 181 (2010), 259-270.

[27] M. Scheffer, S. R. Carpenter. Catastrophic regime shifts in ecosystems: linking theory to observation. Trends Ecol. Evol., 18 (2003), 648-656.

[28] M. Scheffer, J. Bascompte, W. A. Brock, V. Brovkin, S. R. Carpenter, V. Dakos, H. Held, E. H. Van Nes, M. Rietkerk, G. Sugihara. Early-warning signals for critical transitions. Nature, 461 (2009), 53-59.

[29] A. Schmolke, P. Thorbek, D. L. DeAngelis, V. Grimm. Ecological models supporting environmental decision making: a strategy for the future. Trends Ecol. Evol., 25 (2010), 479-486.

[30] M. Schlüter, R. J. J. McAllister, R. Arlinghaus, N. Bunnefeld, K. Eisenack, F. Hölker, E. J. Milner-Gulland, B. Müller, E. Nicholson, M. Quaas, M. Stöven. New horizons for managing the environment: a review of coupled social-ecological systems modeling. Nat. Resour. Model., 25 (2012), 219-272.

[31] R. Seydel. Practical Bifurcation and Stability Analysis, 3rd ed. Springer, New York/Dordrecht Heidelberg, London, 2010.

[32] I. M. Sobol'. Global sensitivity indices for nonlinear mathematical models and their Monte Carlo estimates. Math. Comput. Simul., 55 (2001), 271-280.

[33] I. M. Sobol', S. Kucherenko. Derivative based global sensitivity measures and their link with global sensitivity indices. Math. Comput. Simul., 79 (2009), 3009-3017.

[34] P. A. Stephens, W. J. Sutherland, R. Freckleton. What is the Allee effect? Oikos, 87 (1999), 185-190.

[35] C. M. Taylor, A. Hastings. Allee effects in biological invasions. Ecol. Lett., 8 (2005), 895-908.

[36] G. A. ten Broeke, G. A. K. van Voorn, A. Ligtenberg. Which sensitivity analysis method should I use for my Agent-based Model? JASSS, 19 (2016).

[37] E. H. van Nes, M. Scheffer. Alternative attractors may boost uncertainty and sensitivity in ecological models. Ecol. Model. 159 (2003), 117-124.

[38] G. A. K. van Voorn, L. Hemerik, M. P. Boer, B. W. Kooi. Heteroclinic orbits indicate overexploitation in predator-prey systems with a strong Allee effect. Math. Biosci., 209 (2007), 451-469.

[39] G. A. K. van Voorn, B. W. Kooi, M. P. Boer. Ecological consequences of global bifurcations in some food chain models. Math. Biosci., 226 (2010), 120-133.

[40] B. Walker, C. S. Holling, S. R. Carpenter, A. Kinzig. Resilience, adaptability and transformability in social-ecological systems. Ecol. Soc. 9(2):5. [online] URL: http://www.ecologyandsociety.org/vol9/iss2/art5/ (2004).

[41] S. Wiggins. Introduction to Applied Nonlinear Dynamical Systems and Chaos. Springer, New York, 1990.

\section{A. Direct differential method}

Local sensitivity indices Eqn. (2.1) for ODEs can be estimated using the direct differential method [9,23]. This method expresses each sensitivity index as an additional differential equation to be solved alongside the original ODE model. These equations are obtained by taking the time-derivative of the sensitivity index,

$$
\frac{d}{d t}\left(s_{i, j}\right)=\frac{d}{d t}\left(\frac{\partial Y_{j}}{\partial \theta_{i}}\right)=\frac{\partial}{\partial \theta_{i}}\left(\frac{d Y_{j}}{d t}\right)
$$


with $i=1,2, \ldots, N_{p}$ and $j=1,2, \ldots, N_{v}$.

For ODE models we have analytical expressions for the model equations $\frac{d Y_{j}}{d t}=g_{j}(Y, \theta, t)$, which are inserted in Eqn. (A.1). Considering that $Y_{j}=Y_{j}(\theta, t)$, we use the chain rule for differentiation to rewrite Eqn. (A.1),

$$
\begin{aligned}
\frac{d}{d t}\left(s_{i, j}\right) & =\frac{\partial g_{j}}{\partial \theta_{i}}+\sum_{m=1}^{N_{v}} \frac{\partial g_{j}}{\partial Y_{m}} \frac{\partial Y_{m}}{\partial \theta_{i}} \\
& =\frac{\partial g_{j}}{\partial \theta_{i}}+\sum_{m=1}^{N_{v}} \frac{\partial g_{j}}{\partial Y_{m}} s_{i, m}
\end{aligned}
$$

with the summation running over all state variables. Since at $t=0$ the state variables are given by their initial conditions, we have $s_{i, j}(0)=1$ for the sensitivity to the initial condition of $Y_{j}$, and $s_{i, j}(0)=0$ for other parameters. Eqn. (A.3) is solved numerically alongside the original oDE model to obtain the local sensitivity indices as a function of time.

It can be shown that in the case the original ODE model is stable, the extended system Eqn. (A.3) is also stable. This was shown in Figure 3a, for the nominal point. However, when the original system is unstable and possesses a periodic attractor (in the blue region in Figure 2a) the sensitivity index to the initial condition of $Y_{j}$ shows, just as the original state variables, a periodic behaviour while the other sensitivity indices grow in time oscillatory without bounds when time goes to infinity. Note that the effects of these oscillations are not visible in Figure $4 \mathrm{~b}$ because none of the grid points was in the blue region in Figure 2a.

\section{B. Local sensitivity analysis of the Bazykin-Berezovskaya model}

The local sensitivity indices of the Bazykin-Berezovskaya model Eqn. (3.1) are obtained as a function of time using the direct differential method (Appendix A). Taking the derivative of the model equations Eqn. (3.1) with respect to the parameter $\zeta$, while noting that the state variables are functions of the parameters, yields

$$
\begin{aligned}
\frac{d}{d t}\left(\frac{\partial Y_{1}}{\partial \zeta}\right)= & \frac{\partial Y_{1}}{\partial \zeta}\left(Y_{1}-\zeta\right)\left(\kappa-Y_{1}\right)+Y_{1}\left(\frac{\partial Y_{1}}{\partial \zeta}-1\right)\left(\kappa-Y_{1}\right)- \\
& Y_{1}\left(Y_{1}-\zeta\right) \frac{\partial Y_{1}}{\partial \zeta}-\frac{\partial Y_{1}}{\partial \zeta} Y_{2}-Y_{1} \frac{\partial Y_{2}}{\partial \zeta} \\
\frac{d}{d t}\left(\frac{\partial Y_{2}}{\partial \zeta}\right)= & \gamma \frac{\partial Y_{1}}{\partial \zeta} Y_{2}+\gamma\left(Y_{1}-h\right) \frac{\partial Y_{2}}{\partial \zeta}
\end{aligned}
$$

Similarly, for $h$

$$
\begin{aligned}
\frac{d}{d t}\left(\frac{\partial Y_{1}}{\partial h}\right)= & \frac{\partial Y_{1}}{\partial h}\left(Y_{1}-\zeta\right)\left(\kappa-Y_{1}\right)+Y_{1} \frac{\partial Y_{1}}{\partial h}\left(\kappa-Y_{1}\right)- \\
& Y_{1}\left(Y_{1}-\zeta\right) \frac{\partial Y_{1}}{\partial h}-\frac{\partial Y_{1}}{\partial h} Y_{2}-Y_{1} \frac{\partial Y_{2}}{\partial h} \\
\frac{d}{d t}\left(\frac{\partial Y_{2}}{\partial h}\right)= & \gamma\left(\frac{\partial Y_{1}}{\partial h}-1\right) Y_{2}+\gamma\left(Y_{1}-h\right) \frac{\partial Y_{2}}{\partial h}
\end{aligned}
$$

and for $Y_{2}(0)$ 


$$
\begin{aligned}
\frac{d}{d t}\left(\frac{\partial Y_{1}}{\partial Y_{2}(0)}\right)= & \frac{\partial Y_{1}}{\partial Y_{2}(0)}\left(Y_{1}-\zeta\right)\left(\kappa-Y_{1}\right)+Y_{1} \frac{\partial Y_{1}}{\partial Y_{2}(0)}\left(\kappa-Y_{1}\right)- \\
& Y_{1}\left(Y_{1}-\zeta\right) \frac{\partial Y_{1}}{\partial Y_{2}(0)}-\frac{\partial Y_{1}}{\partial Y_{2}(0)} Y_{2}-Y_{1} \frac{\partial Y_{2}}{\partial Y_{2}(0)} \\
\frac{d}{d t}\left(\frac{\partial Y_{2}}{\partial Y_{2}(0)}\right)= & \gamma \frac{\partial Y_{1}}{\partial Y_{2}(0)} Y_{2}+\gamma\left(Y_{1}-h\right) \frac{\partial Y_{2}}{\partial Y_{2}(0)} .
\end{aligned}
$$

Since the state variables at $t=0$ are given by their initial conditions, the initial conditions of Eqns. (B.1B.5) are equal to zero. For Eqn. (B.6) we have $\frac{\partial Y_{2}(0)}{\partial Y_{2}(0)}=1$. Eqns. (B.1-B.6) are then solved numerically alongside the model equations to obtain the sensitivity indices as a function of time for any given set of parameter values.

\section{Distribution of local sensitivity indices}

The local sensitivity of steady state value $Y_{2}^{*}$ Eqn. (3.2) with respect to model parameter $h$ reads

$$
s_{h}^{*}=\frac{\partial Y_{2}^{*}}{\partial h}=1+\zeta-2 h .
$$

Here we have set all parameters at their nominal values except for $\zeta$ and $h$. Eqn. (C.1) measures the sensitivity around a point in parameter space. To measure the sensitivity over a larger region of parameter space we use the expectation value and variance of Eqn. (C.1) over this region. For the stable steady state region (green) in Figure 2a we have for the expectation value $E\left(s_{h}^{*}\right)$

$$
E\left(s_{h}^{*}\right)=\int_{h=1 / 2}^{h=1} \int_{\zeta=0}^{\zeta=2 h-1} 4 s_{h}^{*} d \zeta d h,
$$

where the factor 4 is a normalisation factor to ensure that the total probability is equal to one. This normalisation factor is equal to one divided by the area of the surface over which we integrate. For the variance $V\left(s_{h}^{*}\right)$ we have

$$
V\left(s_{h}^{*}\right)=\int_{h=1 / 2}^{h=1} \int_{\zeta=0}^{\zeta=2 h-1} 4\left(s_{h}^{*}-E\left(s_{h}^{*}\right)\right)^{2} d \zeta d h .
$$

The local sensitivity of the steady state value $Y_{2}^{*}$ with respect to model parameter $\zeta$ reads

$$
s_{\zeta}^{*}=\frac{\partial Y_{2}^{*}}{\partial \zeta}=h-\kappa
$$

We have for the expectation value $E\left(s_{\zeta}^{*}\right)$

$$
E\left(s_{\zeta}^{*}\right)=\int_{h=1 / 2}^{h=1} \int_{\zeta=0}^{\zeta=2 h-1} 4 s_{\zeta}^{*} d \zeta d h
$$

and for the variance $V\left(s_{\zeta}^{*}\right)$

$$
V\left(s_{\zeta}^{*}\right)=\int_{h=1 / 2}^{h=1} \int_{\zeta=0}^{\zeta=2 h-1} 4\left(s_{\zeta}^{*}-E\left(s_{\zeta}^{*}\right)\right)^{2} d \zeta d h .
$$


Instead of the local sensitivity index of Eqn. (C.1), the expectation value and variance may be computed for the DELSA sensitivity indices, by normalising the partial derivative according to Eqn. (2.9). The calculation of the expectation value and the variance then proceeds in the same way.

\section{Variance decomposition in Sobol' method}

The variance decomposition Eqn. (2.3) follows from a decomposition of the model in terms of the different model parameters $[25,32]$. We write the model as

$$
Y=f(\theta),
$$

where $\theta=\left(\theta_{1}, \ldots, \theta_{N_{p}}\right) \in I^{N_{p}}$ and $I^{N_{p}}$ the $N_{p}$-dimensional unit hypercube. We assume uniform distributions between 0 and 1 for all parameters $\left(\theta_{i} \sim U(0,1)\right)$ and decompose the model output as

$$
f\left(\theta_{1}, \theta_{2}, \ldots, \theta_{N_{p}}\right)=f_{0}+\sum_{i} f_{i}\left(\theta_{i}\right)+\sum_{i<l} f_{i, l}\left(\theta_{i}, \theta_{l}\right)+\ldots+f_{1,2, \ldots N_{p}}\left(\theta_{1}, \theta_{2}, \ldots, \theta_{N_{p}}\right)
$$

The decomposition of Eqn. (D.2) is not unique [25]. If the decomposition is chosen such that all the terms have zero mean,

$$
\int f_{i}\left(\theta_{i}\right) d \theta_{i}=0
$$

then it follows [32] that all the pairs are orthogonal,

$$
\int f_{i}\left(\theta_{i}\right) f_{l}\left(\theta_{l}\right) d \theta_{i} d \theta_{l}=0
$$

Squaring and integrating Eqn. (D.2) then yields the variance decomposition,

$$
\int f(\theta)^{2} d \theta-f_{0}^{2}=\sum_{i} \int f_{i}^{2} d \theta_{i}+\sum_{i<l} \int f_{i, l}^{2} d \theta_{i} d \theta_{l}+\int f_{1,2, \ldots, N_{p}}^{2} d \theta_{1} d \theta_{2} \ldots d \theta_{N_{p}},
$$

or

$$
V\left(Y_{j}\right)=\sum_{i} V_{i}+\sum_{i<l} V_{i, l}+\ldots+V_{1,2, \ldots, N_{p}}
$$

Thus, the total variance is decomposed into terms that can be attributed to (combinations of) model parameters. It follows from Eqn. (D.3) that the terms of Eqn. (D.2) have an interpretation as conditional expectations of the model output,

$$
\begin{aligned}
f_{0} & =E(Y), \\
f_{i} & =E\left(Y \mid \theta_{i}\right)-f_{0}, \\
f_{i, l} & =E\left(Y \mid \theta_{i}, \theta_{l}\right)-f_{i}-f_{l}-f_{0} .
\end{aligned}
$$

The terms of the variance decomposition Eqn. (D.6) can therefore be expressed as

$$
V_{i}=V_{\theta_{i}}\left(E_{\theta_{\sim i}}\left(Y_{j} \mid \theta_{i}\right)\right)
$$

which gives the commonly used measure for the first-order sensitivity index Eqn. (2.6). Expressions for the higher order sensitivity indices are derived in the same way. 


\section{E. Sobol' indices in the steady state}

To illustrate the computation of the Sobol' indices and show the limitations of the methodology in case of parameter dependencies, we calculate here the first- and total-order sensitivity indices Eqns. (2.6-2.7) for the steady state Eqn. (3.2) of the Bazykin-Berezovskaya model. As parameter range for $h$ and $\zeta$ we use the green region of bistability in Figure 2a. We assume that the initial conditions are chosen such that the model evolves to the positive steady state $Y^{*}$ and keep all other parameters fixed at the nominal values. Note that the chosen parameter range imposes a dependency between the parameters $h$ and $\zeta$. As a result the normalisation of the sensitivity indices Eqn. (2.3) does not hold for this example.

The steady state value of $Y_{2}$ is given by Eqn. (3.2). Since the global sensitivity indices are normalised with respect to the total variance, we first calculate the mean and variance of $Y_{2}^{*}$ over the stable steady state region (green) in Figure 2a,

$$
\begin{aligned}
E\left(Y_{2}^{*}\right) & =\int_{\zeta=0}^{\zeta=1} \int_{h=(\zeta+1) / 2}^{h=1} Y_{2}^{*} P_{h}(h) P_{\zeta}(\zeta) d h d \zeta \\
V\left(Y_{2}^{*}\right) & =\int_{\zeta=0}^{\zeta=1} \int_{h=(\zeta+1) / 2}^{h=1}\left(Y_{2}^{*}-E\left(Y_{2}^{*}\right)\right)^{2} P_{h}(h) P_{\zeta}(\zeta) d h d \zeta
\end{aligned}
$$

where the probability density functions $P_{h}=\frac{2}{1-\zeta}$ and $P_{\zeta}=1$ describe a uniform probability density over the region. To obtain the expression for $P_{h}$, note that for any given value of $\zeta$, the length of the interval of $h$ within the stable steady state region is equal to $\frac{1-\zeta}{2}$. The corresponding uniform probability density is given by 1 divided by this length.

To obtain the first-order sensitivity index of $\zeta$ we first compute the expectation value of $Y_{2}^{*}$ while keeping $\zeta$ fixed,

$$
E_{h}\left(Y_{2}^{*} \mid \zeta\right)=\int_{h=(\zeta+1) / 2}^{h=1} Y_{2}^{*} P_{h}(h) d h,
$$

with a subscript to denote the variable over which the expectation value is evaluated. Observe that the expectation $E_{h}\left(Y_{2}^{*} \mid \zeta\right)$ is still a function of $\zeta$. The variance $V_{\zeta}\left(E_{h}\left(Y_{2}^{*} \mid \zeta\right)\right)$ is computed over the range of $\zeta$. We first calculate the mean

$$
E_{\zeta}\left(E_{h}\left(Y_{2}^{*} \mid \zeta\right)\right)=\int_{\zeta=0}^{\zeta=1} E_{h}\left(Y_{2}^{*} \mid \zeta\right) P_{\zeta}(\zeta) d \zeta
$$

and then the variance

$$
V_{\zeta}\left(E_{h}\left(Y_{2}^{*} \mid \zeta\right)\right)=\int_{\zeta=0}^{\zeta=1}\left(E_{h}\left(Y_{2}^{*} \mid \zeta\right)-E_{\zeta}\left(E_{h}\left(Y_{2}^{*} \mid \zeta\right)\right)\right)^{2} P_{\zeta}(\zeta) d \zeta
$$

This expression is normalised with respect to the total variance to obtain the first-order sensitivity index.

Similarly, for the first-order sensitivity of $h$, we have

$$
E_{\zeta}\left(Y_{2}^{*} \mid h\right)=\int_{\zeta=0}^{\zeta=2 h-1} Y_{2}^{*} \hat{P}_{\zeta}(\zeta) d \zeta
$$

with $\hat{P}_{\zeta}=\frac{1}{2 h-1}$ to ensure a uniform distribution over the region. For the variance of this expectation value we then have 


$$
\begin{aligned}
& E_{h}\left(E_{\zeta}\left(Y_{2}^{*} \mid h\right)\right)=\int_{h=\frac{1}{2}}^{h=1} E_{\zeta}\left(Y_{2}^{*} \mid h\right) \hat{P}_{h}(h) d h \\
& V_{h}\left(E_{\zeta}\left(Y_{2}^{*} \mid h\right)\right)=\int_{h=\frac{1}{2}}^{h=1}\left(E_{\zeta}\left(Y_{2}^{*} \mid h\right)-E_{h}\left(E_{\zeta}\left(Y_{2}^{*} \mid h\right)\right)\right)^{2} \hat{P}_{h}(h) d h
\end{aligned}
$$

where $\hat{P}_{h}=2$ is a uniform probability distribution between $h=\frac{1}{2}$ and $h=1$. This expression is inserted into Eqn. (2.6). The derivation of the total-order indices Eqn. (2.7) is not given here, but is similar to the first-order indices.

The resulting values for the first- and total-order sensitivities are given in Table 3 . The outcomes are difficult to interpret because Eqn (2.3) holds only for independent parameters. For dependent parameters, the sensitivity indices are thus not properly normalised, and are not bound between 0 and 1 . In the present case, we find that $h$ has the largest first-order sensitivity index, but the value is larger than one, which is normally not possible since the sensitivity indices are expressed as proportions of the total variance that are explained by a certain parameter. Furthermore, both of the total-order sensitivities are smaller than the corresponding sensitivities, which does not make sense given that the total-order sensitivity includes higher-order effects in addition to first-order effects.

To apply the Sobol' method to the stable steady state region (green) of Figure 2a without violating the assumption of independent parameters, we might perform a parameter transformation that transforms the triangular region into a square region [20]. However, since the sensitivity indices are then computed for the transformed independent parameters instead of the original parameters, it is difficult to interpret the outcomes in terms of the original model parameters. This approach therefore does not really remedy the limitations of the Sobol' method.

Instead of considering only the steady state region, the Sobol' method can be applied to the entire parameter range shown in Figure 2a. The parameters $h$ and $\zeta$ are then independent and ranging between 0 and 1 , thus ensuring proper normalisation of the sensitivity indices. These sensitivity indices cannot be calculated analytically because we have no analytical expression for the model output for the blue region in Figure 2a. This calculation was therefore done numerically using both the factorial design and the design by [26]. The results at $t=10, t=50$, and $t=200$ are given in Table E.1 and are discussed in Section 4.2 .

\section{F. Sampling method for the Sobol' indices}

The methodology described by [26] is a numerical method to estimate Eqns. (2.6-2.7), based on the generation of two sample matrices, A and B. Each contains the same number of sample points $N_{s}$, drawn independently from the probability density functions of the parameters. Then $N_{p}$ recombination matrices $\mathbf{A}_{\mathbf{B}}{ }^{(i)}\left(\mathbf{B}_{\mathbf{A}}{ }^{(i)}\right)$ are generated from the two sampling matrices, where $\mathbf{A}_{\mathbf{B}}{ }^{(i)}\left(\mathbf{B}_{\mathbf{A}}{ }^{(i)}\right)$ is identical to $\mathbf{A}(\mathbf{B})$ except for its $i^{\text {th }}$ column, that is taken from $\mathbf{B}(\mathbf{A})$. The model is then run for each sample point in the sample matrices and the recombination matrices, generating a vector of output values. The computational costs of generating these data is thus $2 N_{s}\left(N_{p}+1\right)$ model runs.

Writing $Y_{A}$ for the vector that contains the output values of sample matrix $A$, the first-order index can be estimated as [26]

$$
S_{i} \approx S_{i}^{A}=\frac{1}{V(Y)}\left(\frac{1}{N_{s}} Y_{A} \cdot Y_{B_{A}}-E(Y)^{2}\right)
$$

where $\cdot$ denotes the inner product and the index $j$ is dropped for convenience. One may also use the alternative 
TABLE E.1. Sample mean, variance, first-order $S_{i}$ and total order $\tilde{S}_{i}$ variance-based sensitivities of $Y_{2}$ at $t=10$ (a), $t=50$ (b), and $t=200$ (c), expressed as percentages. Parameters $Y_{1}(0)$, $\gamma=1$., and $\kappa=1$. are fixed. Parameters $\zeta$ and $h$ are sampled uniformly between 0 and 1., and $Y_{2}(0)$ between 0 and 0.2. First columns: sampling from a $n \times n \times n$ grid, with $n$ indicated. Last columns: using estimators Eqn. (F.3), Eq (F.6), Eqn. (F.10), and Eqn. (F.11) for different $N_{s}$. $C C$ indicates computational costs measured by number of performed model runs.

\begin{tabular}{lllllll}
\hline & $n=10$ & $n=20$ & $n=40$ & $N_{s}=500$ & $N_{s}=10^{3}$ & $N_{s}=2 \cdot 10^{3}$ \\
\hline$C C$ & 1000 & 8000 & 64000 & 4000 & 8000 & 16000 \\
\hline$E\left(Y_{j}\right)$ & 0.189 & 0.191 & 0.192 & 0.217 & 0.218 & 0.227 \\
$V\left(Y_{j}\right)$ & 0.053 & 0.057 & 0.059 & 0.055 & 0.049 & 0.047 \\
$S_{\zeta}$ & 10.72 & 9.395 & 8.874 & 4.307 & 7.301 & 10.08 \\
$S_{h}$ & 92.66 & 88.66 & 86.77 & 68.28 & 74.88 & 88.10 \\
$S_{y_{2}(0)}$ & 2.207 & 1.936 & 1.796 & 1.816 & 2.646 & 3.974 \\
$\tilde{S}_{\zeta}$ & 12.29 & 12.04 & 11.86 & 26.96 & 17.53 & 4.828 \\
$\tilde{S}_{h}$ & 87.84 & 88.84 & 89.24 & 88.43 & 88.31 & 85.70 \\
$\tilde{S}_{y_{2}(0)}$ & 3.666 & 4.295 & 4.410 & 22.05 & 13.82 & 1.589 \\
\hline
\end{tabular}

(a) $t=10$

\begin{tabular}{lllllll}
\hline & $n=10$ & $n=20$ & $n=40$ & $N_{s}=500$ & $N_{s}=10^{3}$ & $N_{s}=2 \cdot 10^{3}$ \\
\hline$C C$ & 1000 & 8000 & 64000 & 4000 & 8000 & 16000 \\
\hline$E\left(Y_{j}\right)$ & .0323 & .0412 & .0450 & .034 & .031 & .034 \\
$V\left(Y_{j}\right)$ & .0030 & .0076 & .0120 & .0053 & .0048 & .0050 \\
$S_{\zeta}$ & 29.73 & 14.74 & 9.960 & 24.45 & 18.33 & 16.62 \\
$S_{h}$ & 39.10 & 68.45 & 77.92 & 42.75 & 37.55 & 39.23 \\
$S_{y_{2}(0)}$ & .102 & .016 & .002 & -.275 & -.247 & -3.084 \\
$\tilde{S}_{\zeta}$ & 64.12 & 34.64 & 23.86 & 60.53 & 62.76 & 59.72 \\
$\tilde{S}_{h}$ & 72.55 & 85.87 & 90.25 & 74.27 & 81.65 & 82.38 \\
$\tilde{S}_{y_{2}(0)}$ & 2.993 & 1.553 & 1.197 & 7.221 & 5.209 & 3.431 \\
\hline
\end{tabular}

(b) $t=50$

\begin{tabular}{lllllll}
\hline & $n=10$ & $n=20$ & $n=40$ & $N_{s}=500$ & $N_{s}=10^{3}$ & $N_{s}=2 \cdot 10^{3}$ \\
\hline$C C$ & 1000 & 8000 & 64000 & 4000 & 8000 & 16000 \\
\hline$E\left(Y_{j}\right)$ & .0288 & .0313 & .0345 & .0276 & .310 & .0292 \\
$V\left(Y_{j}\right)$ & .005 & .0006 & .0006 & .00558 & .00669 & .0625 \\
$S_{\zeta}$ & 33.90 & 33.90 & 31.37 & 19.8 & 17.4 & 15.2 \\
$S_{h}$ & 14.05 & 10.58 & 17.33 & 58.2 & 57.5 & 49.2 \\
$S_{y_{2}(0)}$ & .047 & .121 & .036 & -1.93 & -1.40 & -1.01 \\
$\tilde{S}_{\zeta}$ & 87.04 & 89.56 & 82.93 & 40.7 & 42.2 & 49.0 \\
$\tilde{S}_{h}$ & 69.00 & 67.37 & 64.07 & 78.9 & 84.4 & 83.9 \\
$\tilde{S}_{y_{2}(0)}$ & .928 & 6.407 & 4.442 & 3.25 & -3.66 & 2.25 \\
\hline
\end{tabular}

(c) $t=200$

$$
S_{i} \approx S_{i}^{B}=\frac{1}{V(Y)}\left(\frac{1}{N_{s}} Y_{B} \cdot Y_{A_{B}}-E(Y)^{2}\right),
$$

or the sum of Eqns. (F.1-F.2) divided by two [12]

$$
S_{i} \approx \frac{1}{2}\left(S_{i}^{A}+S_{i}^{B}\right)
$$

The total-order sensitivity indices Eqn. (2.7) are estimated as [26], 


$$
\tilde{S}_{i} \approx \tilde{S}_{i}^{A}=1-\frac{1}{V(Y)}\left(\frac{1}{N_{s}} Y_{A} \cdot Y_{A_{B}}-E(Y)^{2}\right)
$$

or alternatively

$$
\tilde{S}_{i} \approx \tilde{S}_{i}^{B}=1-\frac{1}{V(Y)}\left(\frac{1}{N_{s}} Y_{B} \cdot Y_{B_{A}}-E(Y)^{2}\right)
$$

Also here we follow the line of [12] and combine

$$
\tilde{S}_{i} \approx \frac{1}{2}\left(\tilde{S}_{i}^{A}+\tilde{S}_{i}^{B}\right)
$$

The sample mean $E\left(Y_{j}\right)$ and variance $V\left(Y_{j}\right)$ can be approximated in different ways. In the limit case

$$
E(Y) \approx E\left(Y_{A}\right)
$$

which is a commonly used estimator [32]. One can also use the alternative [26]

$$
E(Y) \approx E\left(Y_{B}\right)
$$

However, for limited sample sizes a better estimator of the sample mean is considered to be [26] the square root of

$$
E(Y)^{2} \approx \frac{1}{N_{s}} Y_{A} \cdot Y_{B}
$$

In line with Eqn. (F.3) proposed by [12] we use the sum of Eqns. (F.7-F.8)

$$
E(Y) \approx \frac{1}{2}\left(E\left(Y_{A}\right)+E\left(Y_{B}\right)\right)
$$

and for the variance

$$
V(Y) \approx \frac{1}{2}\left(V\left(Y_{A}\right)+V\left(Y_{B}\right)\right)
$$

The choice of estimator for sample mean, variance, first and total order sensitivity can lead to different results, as can the selected sampling method. Alternative estimators for $S_{i, j}$ and $\tilde{S}_{i, j}$ are discussed by $[12,26]$, while alternative methods are evaluated by [26]. 